\title{
Kidins220/ARMS is expressed in neuroblastoma tumors and stabilizes neurotrophic signaling in a human neuroblastoma cell line
}

\author{
Danny A. Rogers ${ }^{1,2,3}$ and Nina F. Schor ${ }^{1,2,3}$
}

BACKGROUND: Neurotrophic signaling is an important factor in the survival of developing neurons, and the expression of neurotrophic receptors correlates with prognosis in neuroblastoma. Kinase D-interacting substrate of $220 \mathrm{kDa}$ (Kidins220) associates with neurotrophic receptors and stabilizes them, but the expression and function of Kidins220 in neuroblastoma are unknown.

METHODS: We study Kidins220 expression in human neuroblastoma cell lines and tumor samples by western blotting and microarray analyses. We determine the functional consequences of downregulation of Kidins 220 for response of cell lines to oxidative stress, chemotherapeutic treatment, and neurotrophins using small interfering RNA silencing and by measuring cell survival, signaling, and migration.

RESULTS: Kidins220 is expressed in all neuroblastoma tumors and cell lines studied. Downregulation of Kidins220 leads to attenuation of nerve growth factor (NGF)-induced, but not brain-derived neurotrophic factor (BDNF)-induced, MAPK signaling. However, downregulation of Kidins220 does not alter the response to chemotherapeutic drugs or oxidative stress or affect cellular motility.

CONCLUSION: Kidins220 is expressed in neuroblastoma tumors and stabilizes NGF-induced, but not BDNF-induced, survival signaling in neuroblastoma cell lines.

$\mathbf{N}$ eurotrophic signaling is critical for the normal development and maintenance of the sympathetic nervous system, which arises from neural crest cells early in embryogenesis. Two ligand-receptor pairs, brain-derived neurotrophic factor-tropomyosin receptor kinase B (BDNF-TrkB) and nerve growth factor-tropomyosin receptor kinase A (NGFTrkA), mediate the survival of early neuroblasts and the differentiation into mature neurons. Dysfunction of neurotrophic signaling contributes to the pathogenesis of neuroblastoma, a pediatric tumor of sympathetic precursor cells. The clinical characteristics and current treatment of neuroblastoma were recently reviewed (1). Among other factors, clinical prognosis can be predicted by the presence of $\operatorname{Trk} A$ and $\operatorname{TrkB}$ receptors in the tumor, which also alter the biology of neuroblastoma cell lines in vitro (2). Although many of the signaling pathways of these receptors are known, there are no treatments for neuroblastoma that target this system.

The kinase D-interacting substrate of $220 \mathrm{kDa}$ (Kidins220) is a recently discovered protein highly expressed in developing nervous system and initially shown to be a target for receptor tyrosine kinases in neurons (3). The large multidomain structure of Kidins 220 has subsequently been shown to be involved in neurotrophic and other cytokine signaling $(4,5)$, vesicle formation and transport (6-10), and cytoskeletal regulation during migration $(11,12)$, differentiation $(13,14)$, and plasticity (15). The specificity of Kidins 220 for immature neural cells and its involvement in neurotrophic signaling suggest that Kidins220 may have a role in the biology of neuroblastoma. In this regard, Kidins 220 acts as a scaffold for MAPK signaling cascades, which can be initiated by the binding of neurotrophins to TrkA and TrkB. Neuroblastoma prognosis is positively related to TrkA expression and negatively related to TrkB expression (2).

In addition, neuroblastic tumors have genomic instability that leads to increased copy number of whole or partial chromosomes. The short arm of chromosome 2 (2p) often has increased copy number in neuroblastoma, and two genes in this region, $N m y c$ and $A l k$, have been correlated with poor prognosis (16). The Kidins220 locus on chromosome 2p is near the locations of Nmyc and Alk, so there is an increased copy number of this gene, as well, in many neuroblastomas (17).

There is also evidence that Kidins 220 is expressed and has important functions in normal neural crest cells. Kidins 220 expression in mammalian embryos colocalizes both spatially and temporally with the development of the sympathetic nervous system (3). Kidins 220 knockout causes late fetal death because the neural crest-derived cardiac outflow tracts do not form properly (18).

Kidins220 expression is increased in melanoma, another neural crest tumor, compared with benign skin lesions, and silencing of Kidins 220 causes increased apoptosis in tumor xenografts and melanoma cell lines exposed to oxidative stress. The survival effects of Kidins 220 are blocked by MAPK 
inhibitors, and Kidins220-silenced cells are rescued by the establishment of constitutive MAPK activity (19).

In this study, we establish that Kidins220 is expressed in neuroblastoma cell lines and tumors and examine the effects of Kidins220 downregulation on signaling, migratory behavior, and cell survival in a neuroblastoma cell line.

\section{RESULTS}

Kidins220 Is Expressed in Neuroblastoma Cell Lines and Tumor Samples

Although there are no published reports for neuroblastoma, Kidins220 is expressed in many different tissues and cell lines. Most notably in relation to other neural crest tumors, a rat pheochromocytoma cell line (PC12 cells) has been shown to express Kidins220, and its function in these cells in relation to neurotrophic signaling has been studied extensively $(3,8,20)$. Pheochromocytoma is a neuroendocrine tumor that, like neuroblastoma, has its origin in cells of the sympathetic nervous system lineage.

To qualitatively determine whether Kidins 220 is expressed in neuroblastoma, we tested a group of six human neuroblastoma cell lines for Kidins 220 expression by western blotting. These six lines were compared with PC12 cells, which have been shown previously to express Kidins220 (3). All the six neuroblastoma cell lines express the protein with very little variation in the level of expression (Figure 1a). These also express more Kidins 220 qualitatively than the PC12 line compared with actin.

We next wanted to test the expression in human tumor samples. We used a set of publicly available microarray data $(21,22)$ to look at the expression of Kidins 220 in neuroblastoma tumor samples. Table 1 shows the demographics of the patient samples with regard to patient age, tumor stage, and Nmyc amplification status. The array analysis (Figure $1 \mathrm{~b}$ ) shows high expression of Kidins220 in all samples tested. When compared with other genes that are commonly expressed in neuroblastoma, only GAPDH shows consistent expression with the intensity of Kidins 220 across all samples. It is notable that the neighboring genes on chromosome 2p, Alk and Nmyc, which have been shown to be important in neuroblastoma, are not expressed at high levels in all tumor samples.

\section{MAPK Signaling After NGF Treatment Is Attenuated After Knockdown of Kidins220}

Kidins220 acts as a scaffold to prolong neurotrophic signaling through MAPK signaling cascades in both PC12 cells and neuronal cultures. To check the effects of Kidins 220 knockdown on neurotrophic signaling in neuroblastoma cells, we first tested the response of SH-SY5Y neuroblastoma cells to both NGF and BDNF treatment. SH-SY5Y cells respond to both ligands by increasing phophorylation of ERK, but have differential response kinetics based on the ligand (Figure 2a). BDNF causes an early increase in pERK after addition to the medium, which returns to baseline by $30 \mathrm{~min}$ after treatment. By contrast, NGF causes ERK phosphorylation that persists for up to $30 \mathrm{~min}$, which is shown by the significant difference

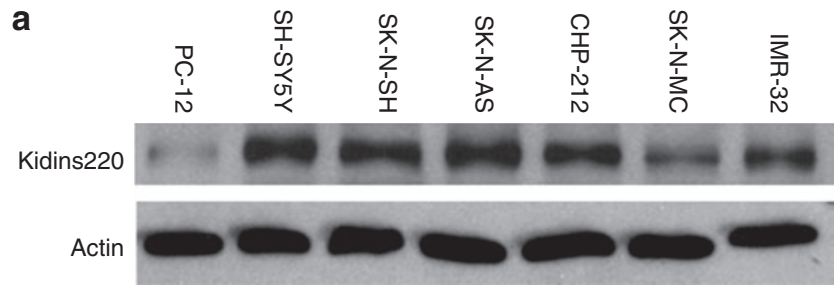

b

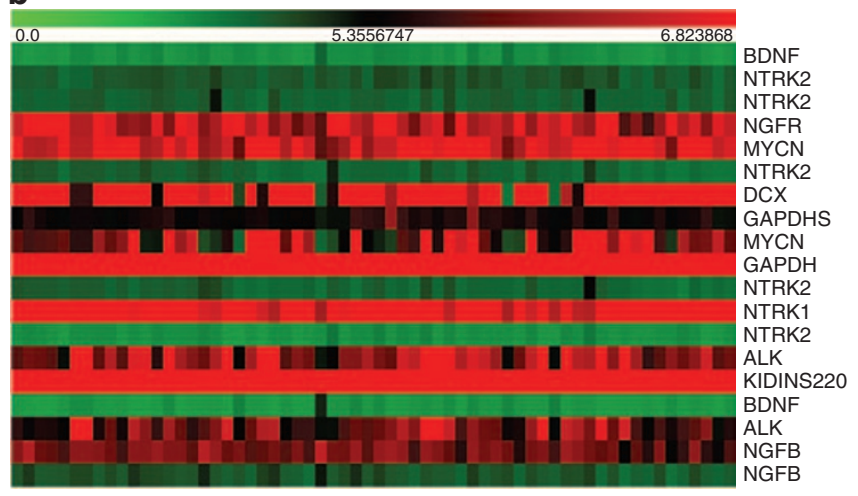

Figure 1. Kinase D-interacting substrate of $220 \mathrm{kDa}$ (Kidins220) is expressed in neuroblastoma cell lines and tumor samples. (a) Western blot from six different neuroblastoma cell lines compared with the PC12 rat pheochromocytoma cell line. Expression is seen in all the cell lines tested. (b) Microarray analysis of neuroblastoma tumor samples assayed for mRNA expression using the Affymetrix U95Av2 whole-genome expression array $(21,22)$. This analysis of 62 samples shows high expression of Kidins 220 across all samples in comparison with other genes that are important in neuroblastoma prognosis. Gene expression is represented on a color scale with green boxes representing low, black boxes representing intermediate, and red boxes representing high expression. Data were normalized with GAPDH representing high expression across all cell lines. Genes are presented in the order they appear on the array. Genes listed more than once are represented by multiple elements on the array.

in $\mathrm{pERK} / \mathrm{ERK}$ ratio with an average difference in three experiments of $0.266 \pm 0.042(P=0.044)$. This prolonged signaling after NGF binding has been ascribed to Kidins220 in other cell types (4). It is interesting to speculate that the absence of prolonged ERK phosphorylation after BDNF treatment may reflect differential affinity of Kidins 220 for TrkA and TrkB receptors in these cells.

We next examined NGF-induced phosphorylation of ERK in Kidins220-small interfering RNA (siRNA)- and nonsilencing scrambled siRNA (NS-siRNA)-transfected cells (Figure $2 \mathbf{b}$ ). Kidins220-siRNA cells show peak ERK phosphorylation at $1 \mathrm{~min}$, with a decrease in phosphorylation thereafter. NS-siRNA cells also peak at $1 \mathrm{~min}$, but have sustained ERK phosphorylation even until $30 \mathrm{~min}$ after treatment (Figure 2c). Significance testing of the quantification of the three independent experiments shows that the average difference in pERK/ERK ratio is $0.326 \pm 0.012, P=0.024$ in NS-siRNA cells at $30 \mathrm{~min}$ after treatment as compared with untreated cells; whereas, there is no difference in Kidins220-siRNA cells between untreated cells and cells harvested $30 \mathrm{~min}$ after treatment (Figure 2d). By contrast, and as one might predict from the already attenuated MAPK signaling in BDNF-treated SH-SY5Y cells, BDNF 
Table 1. Demographics for tumor samples used in microarray analysis

\begin{tabular}{lcc}
\hline & Number of tumors & Percentage of tumors (5) \\
\hline Patient age (mo) & 44 & 71.0 \\
$\leq 18$ & 18 & 29.0 \\
$>18$ & & \\
INSS stage & 4 & 6.5 \\
1 & 10 & 16.1 \\
2 & 9 & 14.5 \\
3 & 30 & 48.4 \\
4 & 9 & 14.5 \\
$4 S$ & 15 & \\
MYCN status & 47 & 24.2 \\
Amplified & & 75.8 \\
Not amplified & 4 & \\
Tumor in sample (\%) & 14 & 6.5 \\
100 & 23 & 22.6 \\
90 & 21 & 37.1 \\
80 & 62 & 33.9 \\
70 & & \\
Total samples & & \\
\hline
\end{tabular}

INSS, International Neuroblastoma Staging System.

signaling is not altered by Kidins220-siRNA transfection (data not shown.).

Cell Culture Growth Rate Under Native, NGF-Enriched, and BDNF-Enriched Conditions Is Unchanged by Downregulation of Kidins220

We next examined the viability of Kidins220-siRNA- and NS-siRNA-transfected SH-SY5Y cells in culture with and without growth factors. After transfection, the cells were starved of growth factors and then treated with growth factor-enriched media for $48 \mathrm{~h}$. Neither NGF nor BDNF treatment altered viability of Kidins220-siRNA- or NS-siRNA-transfected SH-SY5Y cells relative to untransfected controls (Figure 2e).

Kidins220 Downregulation Does Not Affect the Susceptibility of Neuroblastoma Cell Lines to Chemotherapeutic Treatment In Vitro

The current chemotherapeutic treatment for neuroblastoma consists of high-dose antimitotic agents including doxorubicin, etoposide, and vincristine. These cause apoptosis during cell division by intercalating DNA, blocking topoisomerase, or disrupting microtubules, respectively. We investigated whether knockdown of Kidins220 expression would enhance the effects of these drugs in the SH-SY5Y neuroblastoma cell line. Twenty-four hours after transfection with siRNA, cells were treated with one of the chemotherapeutic agents in increasing concentrations. All three drugs caused cell death in a dose-dependent manner; however, Kidins220-siRNA cells exhibited responses that were statistically indistinguishable from NS-siRNA cells (Figure 3).
We also performed viability assays after treatment with the chemotherapeutic agents etoposide, doxorubicin, and vincristine in cells maintained in starvation medium and then supplemented with vehicle or NGF and observed no statistically significant difference between Kidins220-siRNA- and NS-siRNA-transfected cells (see Supplementary Figure S1 online).

\section{Kidins220 Does Not Alter the Response of Neuroblastoma Cell} Lines to Oxidative Injury

In studies of melanoma, another neural crest lineage cancer, Kidins220 depletion was shown to increase apoptosis after oxidative stress caused by exposure to ultraviolet radiation or hydrogen peroxide $\left(\mathrm{H}_{2} \mathrm{O}_{2}\right)(19)$. It has also been shown that neurons of the entorhinal cortex and frontal cortex in heterozygous Kindins 220 knockout mice undergo pyknotic degeneration in a pattern that resembles oxidative damage (23). Neurotrophic signaling pathways have been shown to be involved in oxidative stress responses in neuroblastoma by us and others $(24,25)$, and we considered Kidins 220 as a potential mediator of this response. After transfecting SH-SY5Y cells with Kidins220-specific siRNA or control siRNA, we treated the cells with $150 \mathrm{M} \mathrm{H}_{2} \mathrm{O}_{2}$. We looked at cell survival $24 \mathrm{~h}$ after treatment, and found no significant difference between the Kidins220-siRNA and control groups (Figure 4a).

In melanoma (19), changes in the MAPK signaling cascade were observed after oxidative stress, and this pathway was altered in Kidins220-depleted cells. Specifically, these authors reported that phosphorylation of ERK after ultraviolet exposure was decreased in Kidins220-knockdown cells. We tested the MAPK response to treatment with $150 \mathrm{M} \mathrm{H}_{2} \mathrm{O}_{2}$ in SH-SY5Y cells by looking at phosphorylation of ERK in a time course after treatment (Figure 4b). This study was also performed in the SK-N-SH and CHP-212 human neuroblastoma cell lines (see Supplementary Figure S2 online). Although ERK phosphorylation increases after treatment of neuroblastoma lines with $\mathrm{H}_{2} \mathrm{O}_{2}$, the duration and magnitude of the increase is equivalent in Kidins220-siRNA and NS-siRNA cells. This is shown by the quantification of three independent experiments with no significant difference in the ERK/pERK ratio at each time point (Figure 4c). Most notably, the phosphorylation of ERK is not attenuated at the 30- and 60-min time points in Kidins220-siRNA cells as compared with NS-siRNA cells, suggesting that Kidins220 is not involved in the MAPK/ERK signaling pathway after oxidative stress in these neuroblastoma cells.

Knockdown of Kidins220 Does Not Change Migration Behavior of Neuroblastoma Cells

Cell motility is an important factor in the aggressiveness of neuroblastoma because it determines the capacity of the tumor to form metastases. Kidins 220 is involved in regulating motility in T lymphocytes (12) and dendritic cells in vitro (11) and might control neural progenitor migration as shown by defects in Kidins 220 knockout mice (26). We tested neuroblastoma cell motility in vitro using a simple scratch assay 
a

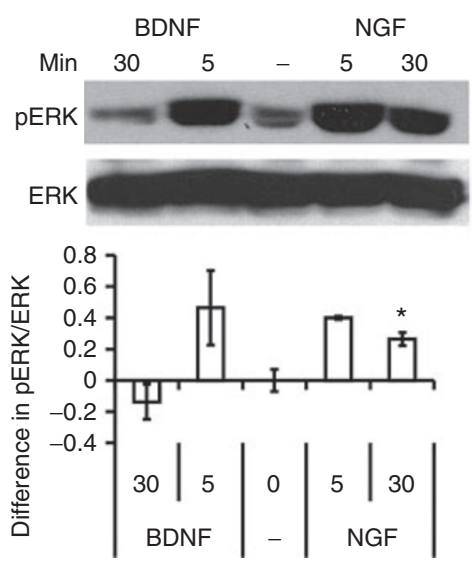

b

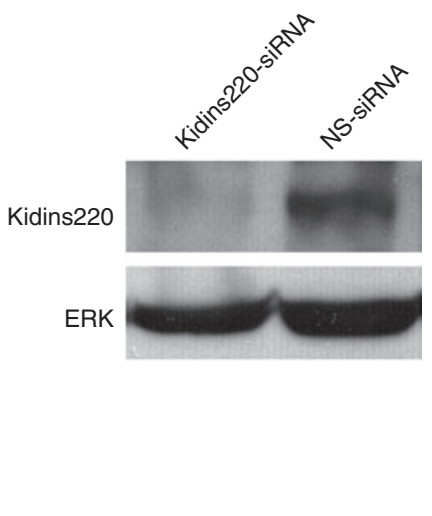

C
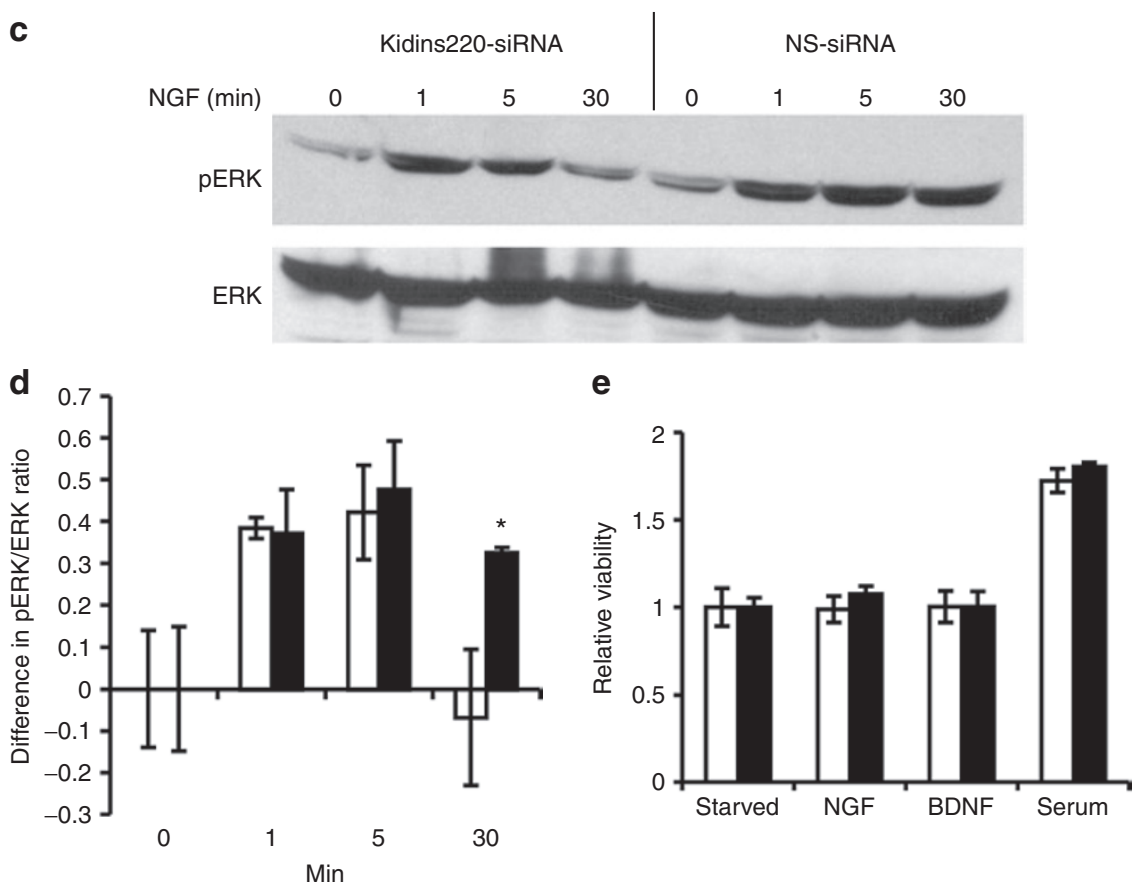

Figure 2. SH-SY5Y cells have attenuated nerve growth factor (NGF) signaling after Kidins 220 knockdown. (a) Representative western blot showing the downstream signaling after treatment of the native SH-SY5Y with neurotrophins. Cells were starved of growth factors overnight and then treated with $50 \mathrm{ng} / \mathrm{ml}$ brain-derived neurotrophic factor (BDNF) or $100 \mathrm{ng} / \mathrm{ml} \mathrm{NGF}$. Cells were harvested either 5 or 30 min after treatment. Accompanying bar graph shows quantification of the difference in pERK/ERK ratio at different time points after treatment. Sample size: $n=3$. Results show that phosphorylation of ERK remains significantly elevated at $30 \mathrm{~min}$ after treatment with NGF; whereas BDNF treatment results in only a short duration increase in ERK phosphorylation with a subsequent return to below baseline. (b) Western blot showing the extent of Kidins220 silencing in this experiment. SH-SY5Y cells were transfected with either Kidins220-specific small interfering RNA (siRNA) duplexes or nonsilencing scrambled (NS) siRNA control duplexes. (c) Transfected cells were then treated with $100 \mathrm{ng} / \mathrm{ml} \mathrm{NGF}$ and harvested after 1, 5, or $30 \mathrm{~min}$ of treatment. Representative western blot shows that phosphorylation of ERK returned to pretreatment levels after $30 \mathrm{~min}$ in the Kidins220-siRNA treated cells. NS siRNA cells showed prolonged phosphorylation of ERK. (d) Quantification of the difference in pERK/ERK ratio after NGF treatment by densitometric analysis of three independent experiments. pERK levels are significantly elevated at $30 \mathrm{~min}$ after treatment in the NS-siRNA group compared with the 0-min timepoint, whereas the level of pERK in Kidins220-siRNA group is not significantly different at $30 \mathrm{~min}$. White bars, Kidins220-siRNA; black bars, NS-siRNA. (e) SH-SY5Y cells are not dependent on neurotrophins for survival or growth. After starvation from growth factors overnight, cells were grown in starvation media supplemented with either $50 \mathrm{ng} / \mathrm{ml} \mathrm{BDNF}$ or $100 \mathrm{ng} / \mathrm{ml} \mathrm{NGF}$. An Alamar Blue viability assay shows that there is no difference in viability after treatment with these growth factors in both the Kidins220-siRNA and NS-siRNA groups. In both cases, addition of serum enhances viable cell number, assumedly because of its positive effect on proliferation. Data represent three independent experiments performed in triplicate. White bars, Kidins220-siRNA; black bars NS-siRNA. In all panels, error bars represent SEM. ${ }^{*} P<0.05$.

where a section of cells is scraped off of the culture dish and then monitored to see whether new cells will migrate into the cleared area (Figure 5a). With serum present in the media, the cells cover the cleared area within $24 \mathrm{~h}$ through migration and proliferation. To reduce proliferation and also test whether neurotrophins influence the migration, we performed the same assay using starvation medium with only $0.5 \%$ serum, or starvation medium plus NGF. In these conditions, there is less proliferation, so the number of cells that migrate into the cleared area more likely reflects migration than proliferation. 

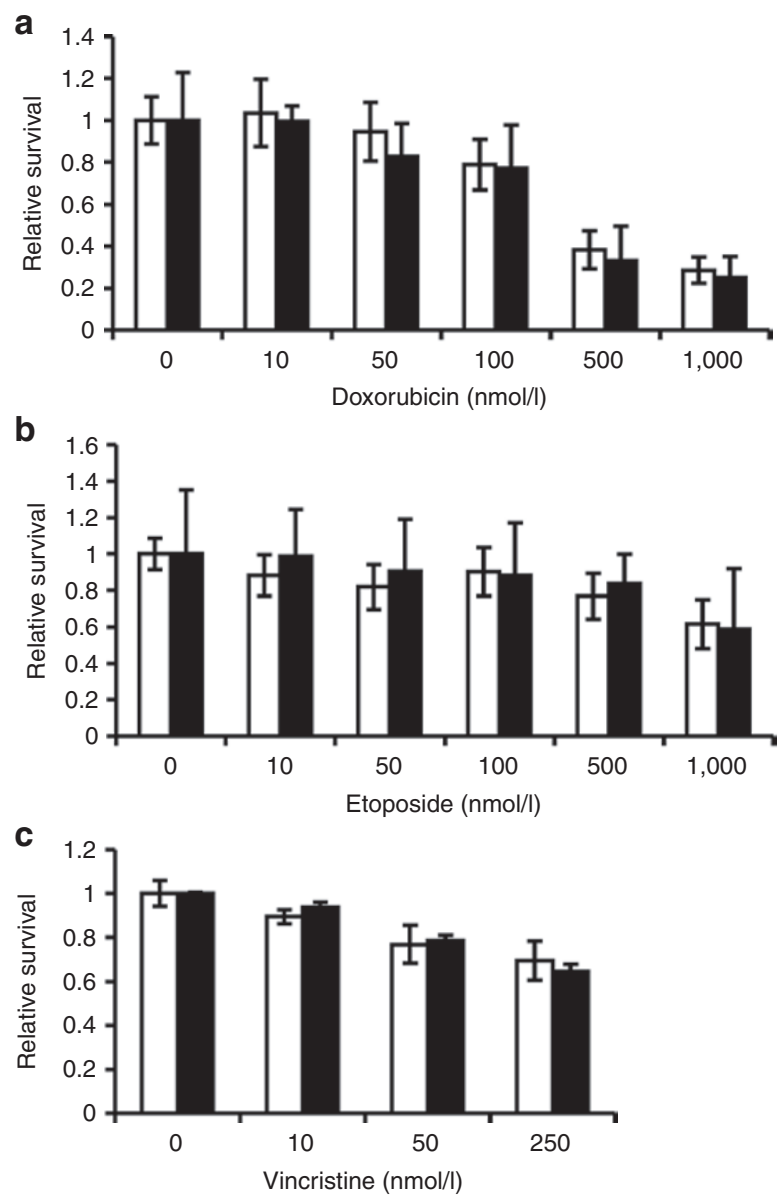

Figure 3. Survival of cultured neuroblastoma cell lines after chemotherapeutic treatment is unaffected by kinase D-interacting substrate of $220 \mathrm{kDa}$ (Kidins220) silencing. SH-SY5Y cells were transfected with Kidins220-siRNA or NS-siRNA. Cells were treated with (a) doxorubicin, (b) etoposide, or (c) vincristine at increasing concentrations. Alamar Blue assay was used $48 \mathrm{~h}$ after drug treatment to assess cell viability. Although these cells are sensitive to all three drugs in a dose-dependent manner, no significant differences were seen in survival between the NS-siRNA and Kidins220-siRNA cells. White bars, NS-siRNA; black bars, Kidins220-siRNA. Data represent two independent experiments performed in quadruplicate samples. Error bars represent SEM. NS, nonsilencing scrambled; siRNA, small interfering RNA.

Quantification of the number of migrating cells per field shows that equal numbers migrate into the cleared area in both Kidins220-siRNA and NS-siRNA cells (Figure $5 \mathbf{b}$ ). There is no difference in the migration ability of SH-SY5Y cells after Kidins220 knockdown.

\section{DISCUSSION}

In this report, we have shown that Kidins 220 is expressed in neuroblastoma patient tumor samples and cell lines. We studied the effects of Kidins220 in the SH-SY5Y cell line and showed that Kidins220 stabilizes the NGF/MAPK/ERK signaling pathway in these cells, but that depleting Kidins220 expression does not alter viability or migration of the cells, the compensatory signaling response or cell viability after treatment with $\mathrm{H}_{2} \mathrm{O}_{2}$, or cell viability after treatment with antimitotic chemotherapeutic drugs. a
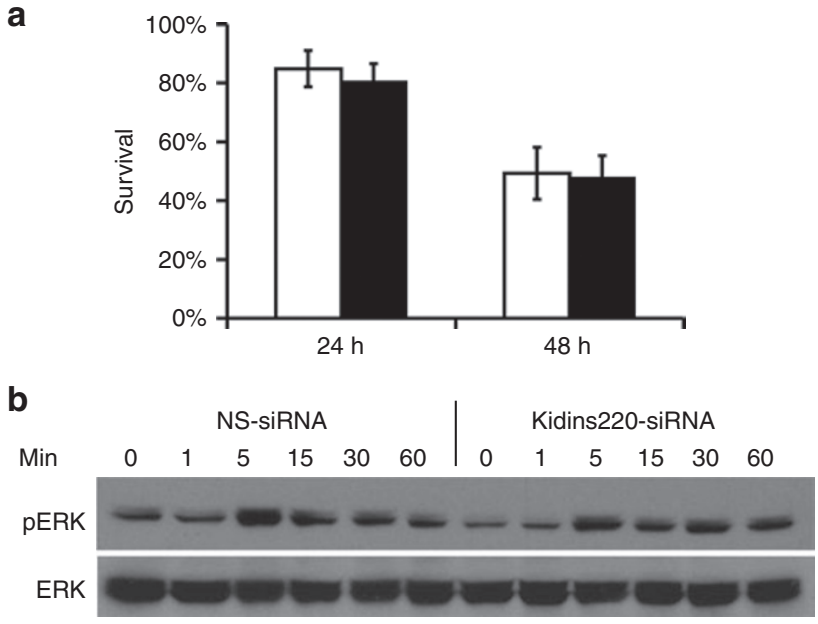

c

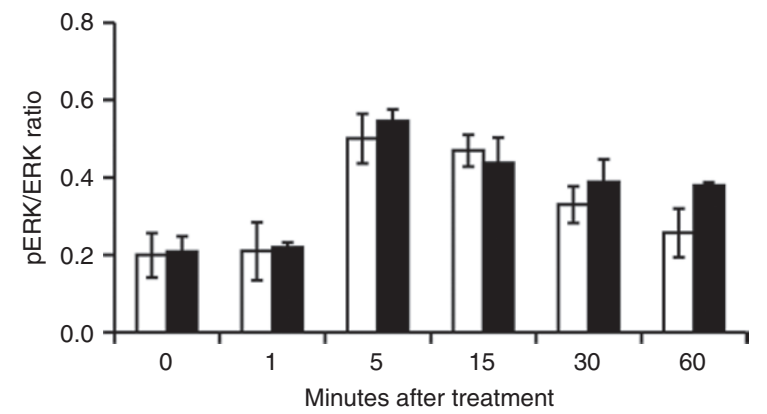

Figure 4. Response of neuroblastoma cells to oxidative stress after kinase D-interacting substrate of $220 \mathrm{kDa}$ (Kidins220) depletion. SH-SY5Y cells were transfected with Kidins220-siRNA or NS-siRNA. After Kidins220 silencing, $\mathrm{SH}-\mathrm{SY} 5 \mathrm{Y}$ cells were treated with $150 \mathrm{~mol} / \mathrm{l}$ hydrogen peroxide $\left(\mathrm{H}_{2} \mathrm{O}_{2}\right)$. (a) 24 and $48 \mathrm{~h}$ after $\mathrm{H}_{2} \mathrm{O}_{2}$ treatment, cells were stained with Trypan Blue and counted. The survival percentage was calculated as the number of cells that are not stained by the dye divided by the total number of cells; $n=3$. Error bars represent SEM. White bars, NS-siRNA; black bars, Kidins220-siRNA. (b) MAPK signaling in response to $\mathrm{H}_{2} \mathrm{O}_{2}$ treatment is unchanged in Kidins220-siRNA cells. Kidins220-siRNA and NS-siRNA cells were treated with $150 \mathrm{~mol} / \mathrm{I} \mathrm{H}_{2} \mathrm{O}_{2}$. Cells were harvested after $1,5,15,30$, or 60 min of treatment. Western blotting shows the survival response of the cell by measuring the phosphorylation of ERK. (c) The quantification of the treatment time course shows that ERK phosphorylation increases 5 min after $\mathrm{H}_{2} \mathrm{O}$ treatment in both the Kidins220-siRNA and NS-siRNA cells. At each time point, there is no significant difference between Kidins220-siRNA and NS-siRNA cells, suggesting that Kidins220 is not involved in ERK signaling in neuroblastoma cells after oxidative stress. Sample size: $n=3$. White bars, NS-siRNA; black bars, Kidins220-siRNA. NS, nonsilencing scrambled; siRNA, small interfering RNA.

We chose to use transient transfection with siRNA to decrease Kidins220 expression. We did this to (i) mimic the effects of a drug with a short half-life that would block Kidins 220 activity and could be given in conjunction with other chemotherapeutics and (ii) minimize compensatory transcriptional changes that would occur as a result of the knockdown. Because of this choice, our results represent only what changes may occur in the $36 \mathrm{~h}$ after blocking Kidins 220 signaling and are not useful in predicting the outcome of neuroblastic tumors that do not express Kidins 220 in their native state.

The efficacy of Kidins 220 knockdown as a potential adjunctive strategy for neuroblastoma treatment hinges on the 


\section{Articles | Rogers and Schor}

a

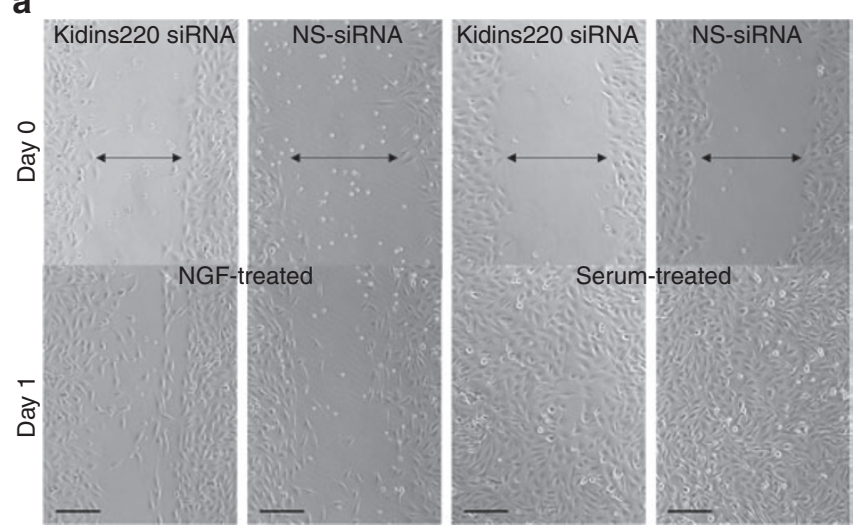

b

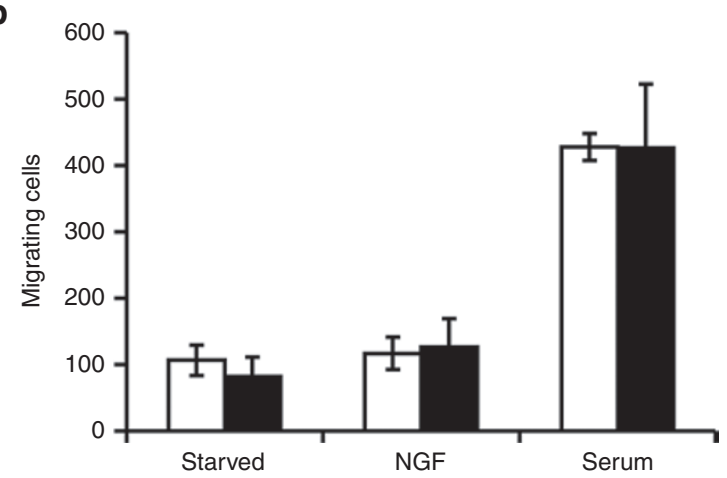

Figure 5. Cell migration is unchanged by Kinase D-interacting substrate of $220 \mathrm{kDa}$ (Kidins220) silencing. SH-SY5Y cells transfected with either Kidins220-siRNA or NS-siRNA were assayed for migration using a scratch assay. (a) Representative bright-field images show plates immediately and $24 \mathrm{~h}$ after scratching. Double-headed arrows designate the area that was cleared by scratching. Nerve growth factor (NGF) cells treated show modest migration into the area on day 1 . Serum-treated cells cover the area through migration and proliferation. (b) Quantification of cells that migrated into the scratched area shows no significant difference between the NS-siRNA groups and the Kidins220-siRNA groups; $n=$ 4. Error bars represent SEM. White bars, Kidins220-siRNA; black bars, NS-siRNA. Scale bars represent $100 \mu \mathrm{m}$. NS, nonsilencing scrambled; siRNA, small interfering RNA.

capability of Kidins220 to promote cell survival by stabilizing MAPK signaling after cellular stress. Liao et al. demonstrated in melanoma that cellular stress caused upregulation of MAPK signaling which was impaired after Kidins220 silencing (19). They proposed that Kidins 220 is a functional part of the main survival pathway. Duffy et al. also showed that a decrease in Kidins220 expression results in cellular damage in the developing mouse brain (23). This damage was not proven to be due to oxidative stress or attributed to a particular pathway, but the damage was phenotypically similar to oxidative damage seen in other studies. In this study, we have shown that Kidins 220 does not affect MAPK signaling induction after treatment with $\mathrm{H}_{2} \mathrm{O}_{2}$, although it affects MAPK signaling after NGF treatment. Therefore, either a separate MAPK signaling pathway is induced by oxidative stress in these cells that does not involve Kidins220 or the MAPK pathway is not the dominant survival pathway in these cells. We have shown previously that p75NTRmediated Akt phosphorylation increases survival after $\mathrm{H}_{2} \mathrm{O}_{2}$ treatment in PC12 cells (24). Although Kidins220 can form complexes that include p75NTR (20), Kidins220 knockdown does not affect Akt signaling in PC12 cells (4). Akt signaling has been shown to be influenced, however, by the effects of Kidins 220 on ephrin signaling. While our work focused on the effects of Kidins 220 on the neurotrophic signaling pathways, Kidins220 associates with ephrin receptors and can modulate ephrin signaling $(3,5,27)$. Ephrins and ephrin receptors have been shown to have increased expression in low-grade tumors that also have high TrkA expression $(28,29)$. In hippocampal neurons from Kidins220-knockout mice, decreased ephrin receptor-modulated phosphatidyl inositol-3-kinase signaling was shown to be associated with a lack of dendrite outgrowth (5). In native SH-SY5Y cells, overexpression of ephrin receptors is associated with growth suppression (29). In our system, we not only expect ephrin receptor expression to be low, but we also assume that knockdown of Kidins 220 would interfere with ephrin signaling. The net result is a decrease in ephrin signaling, which would not change the growth potential of SH-SY5Y cells. This is supported by our results that showed no difference in survival between Kidins220-siRNA and NS-siRNA cells.

In neuroblastoma, blocking Kidins 220 might actually increase cell survival. In PC12 cells, short-lived signaling through the MAPK/ERK signaling cascade leads to proliferation, whereas NGF treatment caused prolonged ERK activation leading to differentiation (30). Our study (Figure 2a) shows that BDNF treatment causes a short-term phosphorylation of ERK in SH-SY5Y cells, and Kidins220 silencing can block the prolonged signaling after NGF treatment, making it analogous to BDNF treatment (Figure 2c). Thus, blocking Kidins220 may cause NGF/TrkA signaling to stimulate proliferation, rather than differentiation, in neuroblastoma cells. This could explain why Kidins220 does not make these cells more susceptible to cell death.

Furthermore, Kidins 220 may differentially associate with TrkA and TrkB receptors, and thereby mediate the differential response of neuroblastoma cells to NGF and BDNF, respectively. This is of clinical interest because TrkA and TrkB expression influence neuroblastoma prognosis in opposite directions (2). A full understanding of the role of Kidins 220 in neuroblastoma clinical behavior must await further studies.

\section{METHODS}

\section{Cell Culture}

The following human neuroblastoma cell lines were obtained from the American Type Culture Collection (Manassas, VA): SK-N-SH, SH-SY5Y, SK-N-AS, SK-N-MC, CHP-212, and IMR-32. PC12 cells were also obtained from the American Type Culture Collection. All cells were grown at $37 \mathrm{C}$ in $5 \% \mathrm{CO}_{2}$. SH-SY5Y cells were cultured in a 1:1 mixture of Dulbecco's Modified Eagle's Medium and Ham's F12 medium (Gibco Life Technologies, Grand Island, NY) supplemented with $10 \% \mathrm{v} / \mathrm{v}$ fetal bovine serum (FBS) (Innovative, Novi, MI), and 1\% $\mathrm{v} / \mathrm{v}$ penicillin/streptomycin (Gibco). PC12 cells were grown in lowglucose Dulbecco's Modified Eagle's Medium (Gibco) supplemented with $10 \% \mathrm{v} / \mathrm{v}$ horse serum (Innovative), $5 \% \mathrm{v} / \mathrm{v}$ FBS, and $1 \% \mathrm{v} / \mathrm{v}$ penicillin/streptomycin (Gibco). All other cell lines were cultured in Eagle’s Minimum Essential Medium (Gibco) supplemented with 1\% $\mathrm{v} / \mathrm{v} 100 \mathrm{mM}$ sodium pyruvate, $1 \% \mathrm{v} / \mathrm{v}$ nonessential amino acids, $10 \%$ $\mathrm{v} / \mathrm{v}$ FBS, and $1 \% \mathrm{v} / \mathrm{v}$ penicillin/streptomycin. Before treatment with 
neurotrophins, SH-SY5Y cells were cultured in starvation medium (Dulbecco's Modified Eagle's Medium :F12 medium supplemented with $0.5 \% \mathrm{v} / \mathrm{v}$ FBS and $1 \% \mathrm{v} / \mathrm{v}$ penicillin/streptomycin).

\section{Western Blotting}

Native neuroblastoma cell lines or transfected and treated cells were washed with ice-cold phosphate-buffered saline (PBS) and lysed in radio immunoprecipitation assay buffer (PBS plus 1\% NP40, $0.5 \%$ sodium deoxycholate, $0.1 \%$ sodium dodecyl sulfate) with proteinase and phosphatase inhibitors. Lysates were then sonicated, and incubated on ice for $30 \mathrm{~min}$, vortexing every $10 \mathrm{~min}$. After centrifugation, the supernatant representing the soluble fraction was isolated. Protein content in the supernatant was analyzed using the Bio-Rad Protein Assay (Bio-Rad, Hercules, CA). Protein loading was normalized based on the quantification. After SDS-polyacrylamide gel electrophoresis, proteins were transferred to a nitrocellulose membrane. Membranes were blocked for nonspecific binding using 5\% w/v nonfat dry milk in PBS for $1 \mathrm{~h}$ at room temperature. Primary antibody staining was performed in 5\% milk in PBS overnight at 4C. The following antibodies and concentrations were used. ARMS/Kidins220-R-300 antibody recognizes C-terminus of Kidins220 (Santa Cruz Biotechnology, Santa Cruz, CA), 1:1000 dilution. This antibody was characterized by comparison with the Kidins220 antibody generated by Maggirwar laboratory (31). In our experiments, it can detect rat, mouse, and human Kidins220; pERK- E-4 antibody recognizes phospho-Tyr 204 in ERK1 and ERK2 (Santa Cruz); ERK- K-23 antibody recognizes ERK1 and ERK2 (Santa Cruz); -actin, 1:5000 (Sigma-Aldrich, St. Louis, MO). Membranes were washed thrice for $5 \mathrm{~min}$ in PBS with $0.05 \%$ Tween-20 (Fisher Scientific, Fair Lawn, NJ). Secondary staining was done with the following horseradish peroxidase-conjugated antibodies in 5\% milk in PBS for $1 \mathrm{~h}$ at room temperature: goat antirabbit immunoglobulin G-horseradish peroxidase, 1:5000 (Santa Cruz); goat antimouse immunoglobulin Ghorseradish peroxidase, 1:5000 (Santa Cruz). Membranes were washed and developed using enhanced chemiluminescence (Santa Cruz).

\section{siRNA Transfection}

Neuroblastoma cell lines were transfected with either a mixture of siRNA oligonucleotides specific for Kidins220 (referred to as Kidins220-siRNA) or NS-siRNA. Both siRNA cocktails were obtained from Santa Cruz Biotechnology. Transfections were performed using Lipofectamine 2000 (Invitrogen, Carlsbad, CA). Cells were plated in appropriate sized plates and allowed to grow overnight. The siRNA oligos were mixed with Lipofectamine reagent in Opti-MEM I medium to form complexes and then added to the cells in media without antibiotics. The final concentration of siRNA in the cultures was $20 \mathrm{nmol} / \mathrm{l}$. The cells were then incubated for $8 \mathrm{~h}$, after which the media were changed and treatments could begin.

\section{Neurotrophin Treatment}

For experiments that required neurotrophin treatment, transfected cells were transitioned to a starvation media $8 \mathrm{~h}$ after transfection. Cells were starved overnight, treated with the desired growth factors, transitioned to standard media with 10\% FBS, or left in the starvation media. All treatments with recombinant human NGF (Peprotech, Rocky Hill, NJ) were done at a concentration of $100 \mathrm{ng} / \mathrm{ml}$. All treatments with recombinant human (Peprotech) were done at $50 \mathrm{ng} / \mathrm{ml}$.

\section{Chemotherapeutic Drug Treatment}

SH-SY5Y cells were plated in 96-well tissue culture dishes at a density of 10,000 cells/well. After transfection with Kidins220-siRNA or NS-siRNA, cells were maintained in the standard media, or starved and treated with growth factors as needed. Stock solutions of etoposide (Sigma), doxorubicin (Toronto Research Chemicals, Toronto, Canada), and vincristine (Sigma) were diluted in the standard media or starvation media to the appropriate concentrations. Cells were treated with the drugs, incubated with for a further $48 \mathrm{~h}$, and then assayed for viability. Cell viability after drug treatments was performed with the Alamar Blue viability assay (Invitrogen). At a specified time after the drug treatment, 10× Alamar Blue reagent was added directly to Kidins220-siRNA or NS-siRNA cells in the 96-well plate to get the proper final concentration. Cells were then incubated for
$1-2 \mathrm{~h}$ at $37^{\circ} \mathrm{C}$. In a Spectromax M5 plate reader (Molecular Devices, Sunnyvale, CA), the fluorescent signal of the reduced dye was excited using $570-\mathrm{nm}$ wavelength light and the emission was read at $585 \mathrm{~nm}$. Relative viability of the cells was determined by comparing the fluorescence of drug-treated cells with that of the untreated controls.

\section{$\mathrm{H}_{2} \mathrm{O}_{2}$ Treatment}

Neuroblastoma cells were cultured in standard media in 24-well tissue culture dishes at a density of 40,000 cells/well. After transfection with siRNA constructs, the cells were treated with a stock solution of $3 \% \mathrm{H}_{2} \mathrm{O}_{2}$ (Sigma) diluted in media to a final concentration of $150 \mathrm{~mol} / \mathrm{l}^{2}$. This solution was immediately added to the cells after making it. For western blotting, the cells were harvested within minutes to hours after treatment. For viability, the cells were incubated for 24 or $48 \mathrm{~h}$ after treatment. Because the Alamar Blue assay is based on a reduction reaction that can be influenced by $\mathrm{H}_{2} \mathrm{O}_{2}$, we used Trypan Blue (Hyclone, Logan, UT) for quantifying survival in the $\mathrm{H}_{2} \mathrm{O}_{2}$ treated cells. At the indicated timepoint after treatment, the cells were trypsinized and collected along with the supernatent to make sure that the nonadherent cells were included in the counts. Suspended cells were mixed with Trypan Blue dye at a 9:1 ratio meaning that the final mixture was $0.04 \%$ Trypan Blue. This mixture was loaded on a hemocytometer and counted to determine the percentage survival.

\section{Migration Assay}

After $24 \mathrm{~h}$, cells were starved of growth factors overnight. A plastic pipette tip was used to scrape away the cells in the middle of the plate. The plates were rinsed and then flooded with media plus $0.5 \%$ FBS, media plus $100 \mathrm{ng} / \mathrm{ml} \mathrm{NGF}$, or media plus $10 \%$ FBS. Images were taken of the cells immediately after scraping and the locations of the images on the plate were marked. After 1 and $2 \mathrm{~d}$, cells were reimaged for determining the extent of cell migration into the scraped area. Quantification was done by tracing the borders of the scraped area in the initial image, and then overlaying the border onto the later images and counting the number of cells within the outline. Cell counts were then normalized based on the area of the outlined section.

\section{Microarray Analysis}

The microarray data used were originally published by Lavarino et al. $(21,22)$, which included a total of 62 unique neuroblastoma samples that were all isolated from adrenal tumors. Data were accessed in the caArray database, which is part of the caBIG project (National Cancer Institute, Bethesda, MD). Normalization of the dataset was done through the Automated Microarray Pipeline using Robust Multiarray Averaging (Dana-Farber Cancer Institute, Boston, MA). The open source MeV experiment viewer (Dana-Farber Cancer Institute) was used to analyze and output the data graphically.

\section{Imaging}

Bright-field images of migrating SH-SY5Y cells were taken on a Nikon Eclipse TE2000-U inverted microscope (Nikon Instruments, Melville, NY) with a SPOT RT Monochrome digital camera and SPOT software (Spot Imaging Solutions, Sterling Heights, MI).

\section{Statistical Analysis}

Significance of quantitative results was determined using an unpaired, two-tailed $t$-test for comparing the groups. In experiments comparing the response after NGF or BDNF treatment, significance was determined by comparing treated cells at a certain time point with untreated cells. In viability experiments comparing Kidins 220 -siRNA with NS-siRNA cells, comparisons were made between groups at the same time point or treatment dose. Statistics were computed using the SPSS 16.0.2 software (SPSS, Chicago, IL).

\section{SUPPLEMENTARY MATERIAL}

Supplementary material is linked to the online version of the paper at http://www.nature.com/pr

\section{STATEMENT OF FINANCIAL SUPPORT}

This study was funded by the William $\mathrm{H}$. Eilinger endowment for pediatric research at the University of Rochester Medical Center and Golisano Children's Hospital, Rochester, NY. 


\section{REFERENCES}

1. Maris JM. Recent advances in neuroblastoma. N Engl J Med 2010;362 2202-11.

2. Schramm A, Schulte JH, Astrahantseff K, et al. Biological effects of TrkA and TrkB receptor signaling in neuroblastoma. Cancer Lett 2005;228: 143-53.

3. Kong H, Boulter J, Weber JL, Lai C, Chao MV. An evolutionarily conserved transmembrane protein that is a novel downstream target of neurotrophin and ephrin receptors. J Neurosci 2001;21:176-85.

4. Arévalo JC, Yano H, Teng KK, Chao MV. A unique pathway for sustained neurotrophin signaling through an ankyrin-rich membrane-spanning protein. EMBO J 2004;23:2358-68.

5. Chen Y, Fu WY, Ip JP, et al. Ankyrin repeat-rich membrane spanning protein (kidins220) is required for neurotrophin and ephrin receptor-dependent dendrite development. J Neurosci 2012;32:8263-9.

6. Bracale A, Cesca F, Neubrand VE, Newsome TP, Way M, Schiavo G. Kidins220/ARMS is transported by a kinesin-1-based mechanism likely to be involved in neuronal differentiation. Mol Biol Cell 2007;18:142-52.

7. Hammond JW, Griffin K, Jih GT, Stuckey J, Verhey KJ. Co-operative versus independent transport of different cargoes by Kinesin-1. Traffic 2008;9:725-41.

8. Hisata S, Sakisaka T, Baba T, et al. Rap1-PDZ-GEF1 interacts with a neurotrophin receptor at late endosomes, leading to sustained activation of Rap1 and ERK and neurite outgrowth. J Cell Biol 2007;178:843-60.

9. Li J, Chen LA, Townsend CM Jr, Evers BM. PKD1, PKD2, and their substrate Kidins 220 regulate neurotensin secretion in the BON human endocrine cell line. J Biol Chem 2008;283:2614-21.

10. Sánchez-Ruiloba L, Cabrera-Poch N, Rodríguez-Martínez M, et al. Protein kinase D intracellular localization and activity control kinase D-interacting substrate of $220-\mathrm{kDa}$ traffic through a postsynaptic density-95/discs large/ zonula occludens-1-binding motif. J Biol Chem 2006;281:18888-900.

11. Riol-Blanco L, Iglesias T, Sánchez-Sánchez N, et al. The neuronal protein Kidins 220 localizes in a raft compartment at the leading edge of motile immature dendritic cells. Eur J Immunol 2004;34:108-18.

12. Jean-Mairet RM, López-Menéndez C, Sánchez-Ruiloba L, et al. The neuronal protein Kidins220/ARMS associates with ICAM-3 and other uropod components and regulates T-cell motility. Eur J Immunol 2011;41: 1035-46.

13. Neubrand VE, Thomas C, Schmidt S, Debant A, Schiavo G. Kidins220/ ARMS regulates Rac1-dependent neurite outgrowth by direct interaction with the RhoGEF Trio. J Cell Sci 2010;123(Pt 12):2111-23.

14. Higuero AM, Sánchez-Ruiloba L, Doglio LE, et al. Kidins220/ARMS modulates the activity of microtubule-regulating proteins and controls neuronal polarity and development. J Biol Chem 2010;285:1343-57.

15. Wu SH, Arévalo JC, Sarti F, Tessarollo L, Gan WB, Chao MV. Ankyrin Repeat-rich Membrane Spanning/Kidins220 protein regulates dendritic branching and spine stability in vivo. Dev Neurobiol 2009;69:547-57.
16. Chen Y, Takita J, Choi YL, et al. Oncogenic mutations of ALK kinase in neuroblastoma. Nature 2008;455:971-4.

17. Stock C, Bozsaky E, Watzinger F, et al. Genes proximal and distal to MYCN are highly expressed in human neuroblastoma as visualized by comparative expressed sequence hybridization. Am J Pathol 2008;172:203-14.

18. Cesca F, Yabe A, Spencer-Dene B, et al. Kidins220/ARMS is an essential modulator of cardiovascular and nervous system development. Cell Death Dis 2011;2:e226.

19. Liao YH, Hsu SM, Huang PH. ARMS depletion facilitates UV irradiation induced apoptotic cell death in melanoma. Cancer Res 2007;67:11547-56.

20. Chang MS, Arevalo JC, Chao MV. Ternary complex with Trk, p75, and an ankyrin-rich membrane spanning protein. J Neurosci Res 2004;78:186-92.

21. Lavarino C, Cheung NK, Garcia I, et al. Specific gene expression profiles and chromosomal abnormalities are associated with infant disseminated neuroblastoma. BMC Cancer 2009;9:44.

22. Lavarino C, Garcia I, Mackintosh C, et al. Differential expression of genes mapping to recurrently abnormal chromosomal regions characterize neuroblastic tumours with distinct ploidy status. BMC Med Genomics $2008 ; 1: 36$

23. Duffy AM, Schaner MJ, Wu SH, et al. A selective role for ARMS/Kidins220 scaffold protein in spatial memory and trophic support of entorhinal and frontal cortical neurons. Exp Neurol 2011;229:409-20.

24. Mi Z, Rogers DA, Mirnics ZK, Schor NF. p75NTR-dependent modulation of cellular handling of reactive oxygen species. J Neurochem 2009;110: 295-306.

25. Tyurina YY, Nylander KD, Mirnics ZK, et al. The intracellular domain of p75NTR as a determinant of cellular reducing potential and response to oxidant stress. Aging Cell 2005;4:187-96.

26. Cesca F, Yabe A, Spencer-Dene B, et al. Kidins220/ARMS mediates the integration of the neurotrophin and VEGF pathways in the vascular and nervous systems. Cell Death Differ 2012;19:194-208.

27. Luo S, Chen Y, Lai KO, et al. \{alpha\}-Syntrophin regulates ARMS localization at the neuromuscular junction and enhances EphA4 signaling in an ARMS-dependent manner. J Cell Biol 2005;169:813-24.

28. Tang XX, Zhao H, Robinson ME, et al. Implications of EPHB6, EFNB2, and EFNB3 expressions in human neuroblastoma. Proc Natl Acad Sci USA 2000;97:10936-41.

29. Tang XX, Evans AE, Zhao H, et al. High-level expression of EPHB6, EFNB2, and EFNB3 is associated with low tumor stage and high TrkA expression in human neuroblastomas. Clin Cancer Res 1999;5:1491-6.

30. Kao S, Jaiswal RK, Kolch W, Landreth GE. Identification of the mechanisms regulating the differential activation of the mapk cascade by epidermal growth factor and nerve growth factor in PC12 cells. J Biol Chem 2001;276:18169-77.

31. Sniderhan LF, Stout A, Lu Y, Chao MV, Maggirwar SB. Ankyrin-rich membrane spanning protein plays a critical role in nuclear factor-kappa B signaling. Mol Cell Neurosci 2008;38:404-16. 